ISSN 0975-3303

Mapana J Sci, 11, 4(2012), 59-64

https:/ / doi.org/ 10.12725/ mjs.23.4

\title{
Antibandwidth of a Graph
}

\author{
Aditya Shastry* and Nidhi Khandelwal ${ }^{\dagger}$
}

\begin{abstract}
The antibandwidth problem consists of placing the vertices of a graph on a line in consecutive integer points in such a way that the minimum difference of adjacent vertices is maximized. This problem is NP- hard. In this paper, we find some bounds for antibandwidth using some invariants of graphs. We prove that considerating the interior boundary and the exterior boundary when estimating the antibandwidth of connected graphs gives the same results.
\end{abstract}

Keywords: Vertex independent number, chromatic number, vertex connectivity, antibandwidth.

\section{Introduction}

Let $G=(V, E)$ be a $(p, q)$-graph. A $1-1$ mapping $f: V(G) \rightarrow\{1,2, \ldots, p\}$ is called a proper numbering of $G$. The antibandwidth $a b(G, f)$ of a proper numbering $f$ of $G$ is the number $a b(G, f)=\min \{|f(u)-f(v)|: u v \in E(G)\}$ and the antibandwidth $a b(G)$ of $G$ is the number $a b(G)=\max \{a b(G, f): f$ is a proper numbering of $G\}$ A proper numbering $f$ is called an antibandwidth numbering of $G$ if $a b(G, f)=a b(G)$.

The antibandwidth problem is a special case of the antidilation problem [1]. Antidilation is a problem of injective embedding of a

* Department of Mathematics and Statistics, Banasthali University, Banasthali -304022, Rajasthan, India, adityashastri@yahoo.com

† Department of Mathematics and Statistics, Banasthali University, Banasthali -304022, Rajasthan, India, nidhi.khandelwal20@gmail.com

Received: July 2012, Reviewed: Aug. 2012 
graph $G$ in a graph $H$ such that the length of the shortest edge of $G$ measured in $H$ is maximized over all possible embeddings of $G$ in $H$. Antidilation is a dual problem to a well studied dilation problem [2]. The special case of dilation is bandwidth problem. The antibandwidth problem is a dual modification of bandwidth problem. Antibandwidth is known as separation number, dual bandwidth as well. Antibandwidth of a graph can be visualized as placing the vertices of graph into the integer points along the $X$ - axis so that length of shortest edge is maximized.

The concept of antibandwidth was introduced by Leung, Vornberger and Witthoff under the name separation number [3]. There was also another name used for this problem. Lin \& Yuan called it dual bandwidth [4]. Leung, Vornberger and Witthoff show by reduction that directed version of antibandwidth problem is connected to some multiprocessor scheduling problems. They show for a graph $G=(V, E)$, it is NP- complete to decide whether $a b(G) \geq 2$. Answering this question is equivalent to finding the Hamiltonian path in the complement of $G$.

A cut set of a connected graph $G$ is a set of vertices whose removal renders $G$ disconnected. The connectivity or vertex connectivity $\kappa(G)$ (where $G$ is not complete graph) is the size of smallest vertex cut set. An independent set or stable set is a set of vertices in a graph, no two of which are adjacent. Vertex independent number $\beta_{0}$ is the cardinality of the largest vertex independent set. The chromatic number of a graph $G$ is the minimum number of colors $\chi(G)$ needed to color the vertices of $G$ so that no two adjacent vertices share the same color.

\section{Some Bounds for Antibandwidth of a Graph}

Our notation follows Harary [5]. Particularly, we reserve: a letter $p$ for number of vertices, $q$ for number of edges, $\Delta$ for the highest degree, $\kappa$ for connectivity, $\beta_{0}$ for vertex independent number, $\chi$ for chromatic number, $d_{i}$ for degrees of vertices $\left(d_{1} \leq d_{2} \leq d_{3} \leq \ldots \leq d_{p}\right)$. 
As in [5], we write $P_{p}^{k}$ for the $k^{\text {th }}$ power of the path $P_{p}$, in which two vertices $\mathrm{u}, \mathrm{v}$ of $V\left(P_{p}\right)=\{1,2, \ldots, p\}$ are adjacent if, and only if $0<|u-v| \leq k$.

Theorem 1. $a b(G)$ is the largest integer $k$ such that $G \subseteq \overline{P_{p}^{k-1}}$.

Proof. Every inclusion $f: V(G) \rightarrow \overline{P_{p}^{k-1}}$ induces a valuation $f: V(G) \rightarrow\{1,2, \ldots, p\}$ such that $\min _{u v \in E(G)}|f(u)-f(v)| \geq k$ and vice versa. Hence $a b(G) \geq k$ if and only if $G \subseteq \overline{P_{p}^{k-1}}$.

Therefore $a b(G)=\max \left\{k: G \subseteq \overline{P_{p}^{k-1}}\right\}$.

Lemma 1. The invariants of the graph $\overline{P_{p}^{k-1}}$ satisfy:

1.

$$
\begin{aligned}
q=\frac{p(p-1)}{2}- & {[(p-1)+(p-2)+\ldots+(p-k+1)] } \\
& =\frac{p(p-1)}{2}-\left[(k-1) p-\frac{k(k-1)}{2}\right]
\end{aligned}
$$

2. $\Delta=p-k$

3. $\beta_{0}=k$

4. $\kappa=\left\{\begin{array}{c}p-2 k+1 \text { if } p>2 k-1 \\ 0 \quad \text { otherwise }\end{array}\right.$

5. $\chi=\left\lceil\frac{p}{k}\right\rceil=\left\{\begin{array}{ll}\frac{p}{k}+1 & \text { if } p \neq 0(\bmod k) \\ \frac{p}{k} & \text { if } p=0(\bmod k)\end{array} \quad \Rightarrow \quad \chi \leq \frac{p}{k}+1\right.$

6. $d_{i}=p-\left[k+\min \left\{\left\lfloor\frac{i-1}{2}\right\rfloor, k-1, p-k\right\}\right], 1 \leq i \leq p$.

Theorem 2. For any connected graph $G$ with $a b(G)=k$, we have

1. $k \geq \frac{1+2 p+\sqrt{8 q+1}}{2}$ or $k \leq \frac{1+2 p-\sqrt{8 q+1}}{2}$

2. $k \leq p-\Delta$ 
3. $k \leq \beta_{0}$

4. $k \leq \frac{p-\kappa+1}{2}$

5. $k \leq \frac{p}{\chi-1}$

6. $k \leq \min _{1 \leq i \leq p} \max \left\{p-d_{i}-\left\lfloor\frac{i-1}{2}\right\rfloor, \frac{p-d_{i}+1}{2}\right\}$.

Proof. By Theorem 1, $G \subseteq \overline{P_{p}^{k-1}}$. Now since $p\left(G_{l}\right)=p\left(G_{2}\right), \quad G_{1} \subseteq G_{2}$ implies $q\left(G_{1}\right) \leq q\left(G_{2}\right), \quad \Delta\left(G_{I}\right) \leq \Delta\left(G_{2}\right), \quad \beta_{0}\left(G_{I}\right) \geq \beta_{0}\left(G_{2}\right), \quad \kappa\left(G_{I}\right) \leq \kappa\left(G_{2}\right)$, $\chi\left(G_{1}\right) \leq \chi\left(G_{2}\right), d_{i}\left(G_{1}\right) \leq d_{i}\left(G_{2}\right)$ for each $i$.

The rest follows by lemma 1 .

Definition. For a subset $S \subseteq V(G)$, the interior and exterior boundaries of $S$ are defined respectively as

$$
\partial(S)=\{u \in S: \exists v \in V(G)-S \text { such that } u v \in E(G)\}
$$

and $N(S)=\{v \in V(G)-S: \exists u \in S$ such that $u v \in E(G)\}$.

Suppose an edge labeling $f$ is given. Let $v_{i}=f^{-1}(i), 1 \leq i \leq p$, define $S_{k}(f)=\left\{v_{p}, v_{p-1}, \ldots, v_{p-k+1}\right\}=f^{-1}\{p, p-1, \ldots, p-k+1\}$.

Theorem 3. Let $G=(V, E)$ be a connected $(p, q)$ - graph. Then $a b(G) \leq \max _{f} \min _{1 \leq k \leq p}\left\{p-\mid \partial\left(S_{k}(f)\right)\right\}$, where $f: V(G) \rightarrow\{1,2, \ldots, p\} \quad$ is an injective mapping.

Proof. Let $f$ be any injective vertex labeling of $G$. Choose a vertex $u \in \partial\left(S_{k}(f)\right)$ such that $f(u)=\min \left\{f(v): v \in \partial\left(S_{k}(f)\right)\right\}$.

Then $p-k+1 \leq f(u) \leq p+1-\left|\partial\left(S_{k}(f)\right)\right|$. Also $f(v) \geq 1 \quad \forall v \in N\left(S_{k}(f)\right)$.

$$
\begin{aligned}
a b(G, f)= & \min \{|f(u)-f(v)|: u v \in E(G)\} \\
& \leq|f(u)-f(v)| \leq|p+1-| \partial\left(S_{k}(f)\right)|-l|=p-\mid \partial\left(S_{k}(f)||\right.
\end{aligned}
$$

Therefore $a b(G) \leq \max _{f} \min _{1 \leq k \leq p}\left\{p-\left|\partial\left(S_{k}(f)\right)\right|\right\}$. 
Theorem 4. Let $G=(V, E)$ be a connected $(p, q)$ - graph. Then $\left.\max _{f} \min _{1 \leq k \leq p}\left\{p-\mid \partial\left(S_{k}(f)\right)\right\}\right\}=\max _{f} \min _{I \leq k \leq p}\left\{p-\left|N\left(S_{k}(f)\right)\right|\right\}$, where the minimum is taken over all vertex labelings of $G$.

Proof. Let $f$ is any vertex labeling of $G$. For $1 \leq k \leq p$, consider the set $S_{k}^{*}(f)$, which is defined as $S_{k}^{*}(f)=V(G)-S_{k}(f), \forall k=1,2, \ldots, p$. Then $\partial\left(S_{k}(f)\right)=N\left(S_{k}^{*}(f)\right) \quad \forall k=1,2, \ldots, p$.

Define a vertex labeling $g$ on $G$ by $S_{k}(g)=S_{p-k}^{*}(f) ; \forall k=1,2, \ldots, p-1$ and $S_{p}(G)=V(G)$. Since $g$ depends uniquely on $f$ and labeling runs over all possible choices, we have

$$
\begin{aligned}
& \min _{l \leq k \leq p}\left\{p-\mid \partial\left(S_{k}(f) \mid\right\}=\min _{1 \leq k \leq p}\left\{p-\left|N\left(S_{k}^{*}(f)\right)\right|\right\}=\min _{1 \leq k \leq p}\left\{p-\left|N\left(S_{k}(g)\right)\right|\right\}\right. \\
& \Rightarrow \quad \max _{f} \min _{1 \leq k \leq p}\left\{p-\left|\partial\left(S_{k}(f)\right)\right|\right\}=\max _{f} \min _{1 \leq k \leq p}\left\{p-\left|N\left(S_{k}(f)\right)\right|\right\} .
\end{aligned}
$$

Corollary 1. Let $G=(V, E)$ be a connected $(p, q)-$ graph. Then $a b(G) \leq \max _{f} \min _{1 \leq k \leq p}\left\{p-\mid N\left(S_{k}(f) \mid\right\}\right.$, where the minimum is taken over all vertex labeling.

\section{Some Applications}

1. Given $n$ transmitters and $n$ frequencies find such a bijective frequency assignment where the interfering transmitters have as different frequency as possible.

Graph Model:

$>$ Transmitters $=$ Vertices; Interferences $=$ Edges between interfering transmitters; Frequency assignment $=$ Optimal antibandwidth labeling.

2. Find a scheduling of a tournament such that every player will rest as much as possible before playing the next match without delaying the whole schedule.

Graph Model: 
$>$ Players = Vertices; Match to be played = Edge; Example: Round-robin tournament $=$ Complete graph; Optimal schedule $=$ Edge antibandwidth of underlying graph .

3. Find a scheduling of a examination in a university such that every student will get holidays as much as possible before giving the next exam without delaying the whole schedule.

Graph Model:

$>$ Examinations $=$ Vertices; Examinations of same class $=$ Edge

$>$ Optimal schedule $=$ Optimal antibandwidth labeling .

4. The problem also belongs to the family of obnoxious facility location problem. The "enemy graph" is representing some kind of "enemy" facilities and the task is to arrange them on the line such that the minimal distance between any of two enemies is maximized. The "enemy" relation is represented by adjacency in the underlying graph.

\section{Conclusion}

In this paper, we find some bounds for antibandwidth using the invariants of the graph $\overline{P_{p}^{k-1}}$. We also find some upper bounds using the interior and exterior boundaries.

\section{References}

[1] Z Miller and D Pritikin, On the separation number of graphs, Networks, vol. 19, pp. 651-666, 1989.

[2] F T Leighton, B M Maggs and S B Rao, Packet routing and job-shop scheduling in $\mathrm{O}$ (congestion + Dilation) steps, Combinatorica, vol. 14, pp. 167-180, 1994.

[3] J Y-T Leung, O Vornberger and J D Witthoff, On some variants of the bandwidth minimization problem, SIAM Journal on Computing, vol. 13, pp. 650-667, 1984.

[4] Y Lin and J Yuan, "The dual bandwidth problem for graphs," Journal of Zhengzhou University, vol. 35, 2003.

[5] F Harary, Graph theory, Massachusetts: Addison-Wesley, 1969. 